\title{
An effective range of polydeoxyribonucleotides is critical for wound healing quality
}

\author{
KYU-HEE HWANG ${ }^{1-3^{*}}$, JI-HEE KIM $^{1-3^{*}}$, EUN YOUNG PARK ${ }^{4 *}$ and SEUNG-KUY CHA ${ }^{1-3,5}$ \\ Departments of ${ }^{1}$ Physiology and ${ }^{2}$ Global Medical Sciences; ${ }^{3}$ Mitohormesis Research Center; \\ ${ }^{4}$ Department of Obstetrics and Gynecology; ${ }^{5}$ Institute of Lifestyle Medicine; \\ Yonsei University Wonju College of Medicine, Wonju, Gangwon-do 26426, Republic of Korea
}

Received May 9, 2018; Accepted August 9, 2018

DOI: $10.3892 / \mathrm{mmr} .2018 .9539$

\begin{abstract}
Wound healing is a physiological restorative response to tissue and cell injury. This process occurs in collaboration with a complex cascade of cellular events, including biochemical alterations to the extracellular matrix. Polydeoxyribonucleotide (PDRN) is a fragmented DNA mixture from Oncorhynchus mykiss or Oncorhynchus keta sperm known to promote tissue regeneration under different pathophysiological conditions. However, the most effective molecular size of PDRNs for promoting the wound healing process and quality has not been established. In the present study, the regeneration quality with low $(<50 \mathrm{kDa})$, middle [classic PDRN; 50-1,500 kDa] and high (>1,500 kDa) molecular weight PDRNs in a skin wound healing mouse model was examined using hematoxylin and eosin, as well as Masson's trichrome stain. A $4 \mathrm{~mm}$ biopsy punch was used to produce wounds in the skin of the mice. PDRN-mediated cellular behavior and signaling were evaluated by in vitro scratch assay and western blot analysis, respectively. It was observed that the apparent surface wound healing processes were not significantly different between PDRN molecular sizes. Immunohistochemical analysis revealed that classic PDRN-injected mice exhibited less lipid accumulation with increased collagen composition. These results suggested that 50-1,500 kDa PDRN offers an effective DNA mixture to improve wound healing quality. Furthermore, classic PDRN increased cell migration via c-Jun $\mathrm{N}$-terminal kinase signaling in human fibroblasts. The present study suggests an optimal PDRN molecular weight to promote wound healing, and
\end{abstract}

Correspondence to: Professor Seung-Kuy Cha, Department of Physiology, Yonsei University Wonju College of Medicine, 20 Ilsan Road, Wonju, Gangwon-do 26426, Republic of Korea

E-mail: skcha@yonsei.ac.kr

*Contributed equally

Key words: polydeoxyribonucleotide, wound healing, skin repair, regeneration, migration novel approaches for therapeutic strategies to improve tissue regeneration quality.

\section{Introduction}

Wound healing is a dynamic and coordinated process consisting of a sequence of cell and tissue repair that is promoted by multiple growth factors, including vascular endothelial growth factor (VEGF), endothelial growth factor (EGF), fibroblast growth factor and platelet-derived growth factor (PDGF) $(1,2)$. The human wound healing process may be divided into three or four distinct phases in a coordinated series of events that includes inflammation, proliferation and extracellular matrix (ECM) remodeling (3). In addition, neoangiogenesis, re-epithelization and the production of novel ECM are vital to the wound healing process (4).

Polydeoxyribonucleotide (PDRN) is an Oncorhynchus mykiss (salmon trout) or Oncorhynchus keta (chum salmon) sperm DNA polymer with a specific molecular weight between 50 and 1,500 kDa with biological therapeutic activity (5). This molecular weight range of PDRN [classic or conventional PDRN] has been applied to promote tissue regeneration in various pathological conditions (6-8). The final PDRN products are produced by controlled processes that may contain sources of purine and pyrimidine deoxynucleosides/deoxynucleotides. The classic PDRN molecular weight of $\sim 132 \mathrm{kDa}$ is formed from purification and sterilization processes $(5,9)$.

Classic PDRN is an $\mathrm{A}_{2 \mathrm{~A}}$ receptor agonist $(10,11)$. Multiple in vitro and in vivo studies demonstrated that PDRN serves as an anti-inflammatory and tissue-regenerating agent. Treatment with classic PDRN significantly improved clinical symptoms by perturbing cytokine signaling in a collagen-induced osteoarthritis model. Classic PDRN upregulates VEGF, collagen and cellular tumor antigen p53; it functions as a negative regulator for tumor necrosis factor- $\alpha$, high mobility group protein B1 and metalloproteinase in tissue regeneration $(7,9)$. Notably, previous clinical studies have demonstrated that PDRN promotes rapid autologous skin graft healing and corneal epithelium regeneration following photorefractive keratectomy, and alleviates pain and disability in patients with rotator cuff disease (12-14). Furthermore, classic PDRN improves wound closure and promotes re-epithelialization in patients with refractory diabetic foot ulcers (15). 
Accumulating evidence suggests that PDRN is a safe and immune response-free substance in multiple clinical settings $(15,16)$. A recent study reported that $O$. keta- and O. mykiss-derived PDRN injections had the same wound healing effects in full-thickness animal models (17). However, the optimal molecular weight of PDRNs for improving the quality and efficacy of wound regeneration has not been determined. In the present study, it was examined whether PDRNs across a range of sizes promoted healing following experimental injury, and whether this effect results from migration processes.

\section{Materials and methods}

Animal model. The experimental protocol used in the present study, including the use of animals, was approved by the Institutional Animal Care and Use Committee, Yonsei University Wonju College of Medicine (Wonju, Korea; approval no. YWC-160504-1). Male hairless mice (SHK1; n=32) aged 8 weeks and weighing 25-30 g were obtained from Orient Bio, Inc. (Seongnam, Korea). Mice were randomly divided into four groups: Control ( $\mathrm{n}=8)$; low (low-PDRN; <50 kDa; $\mathrm{n}=8)$; middle (classic PDRN; 50-1,500 kDa; $\mathrm{n}=8$ ); and high (high-PDRN; $>1,500 \mathrm{kDa} ; \mathrm{n}=8$ ) molecular weight PDRNs. All PDRN products were obtained from Pharma Research Products Co., Ltd. (Pangyo, Korea). All animals were provided with standard laboratory food and water ad libitum. The mice were raised at a constant temperature $\left(22 \pm 3^{\circ} \mathrm{C}\right)$ and relative humidity $(50 \pm 10 \%)$ in a $12 \mathrm{~h}$ light/dark cycle. Wound production was performed under general anesthetic isoflurane inhalation. Under aseptic conditions, a $4 \mathrm{~mm}$ biopsy punch (Kai Europe $\mathrm{GmbH}$, Solingen, Germany) was used to produce wounds in the skin of the mice. Following the procedure, mice were treated with a daily intraperitoneal (i.p.) administration of PDRNs at a dose of $8 \mathrm{mg} / \mathrm{kg}$ (day 0 to 6). The control group received i.p. injection of vehicle $(0.9 \% \mathrm{NaCl})$ at the same volume and time as the treatment group.

Cell culture. A stable human fibroblast cell line (CCD-986SK; Korean Cell Line Bank, Seoul, Korea) was cultured in $10 \%$ fetal bovine serum (FBS; Gibco; Thermo Fisher Scientific, Inc.), 1\% penicillin and streptomycin in high glucose Dulbecco's modified Eagle's medium (DMEM; Hyclone; GE Healthcare Life Sciences, Logan, UT, USA) in a $37^{\circ} \mathrm{C}$ humidified atmosphere of $5 \% \mathrm{CO}_{2}$.

In vitro scratch assay. The human fibroblast cells were seeded at $1 \times 10^{6}$ cells/well in a 6 -well plate and grown to full confluence. Cells were scratched with a $200 \mu 1$ pipette tip across the center of the wells. In order to distinguish cell migration from proliferation, all wound-healing assays were performed with or without anti-tumor drug mitomycin C (cat. no. M4287; Sigma-Aldrich; Merck KGaA, Darmstadt, Germany) treatment, at a final concentration of $5 \mu \mathrm{g} / \mathrm{ml}$. Images were captured using a light microscope (x100), $24 \mathrm{~h}$ after treatment at $37^{\circ} \mathrm{C}$ with the drug (time 0 was the initial time point). The healing area (\%) was measured using Image $\mathbf{J}$ software version 1.8 (National Institutes of Health, Bethesda, MD, USA).

Western blotting. Cells were incubated in 1\% FBS and $1 \%$ antibiotics in high glucose DMEM for $48 \mathrm{~h}$, treated at $37^{\circ} \mathrm{C}$ with PDRNs $(50 \mu \mathrm{g} / \mathrm{ml})$ or EGF ( $30 \mathrm{ng} / \mathrm{ml})$ for $24 \mathrm{~h}$, and subsequently washed with PBS and lysed with radioimmunoprecipitation assay buffer (Intron Biotechnology, Inc., Seongnam, Korea) containing protease and phosphatase inhibitors (Roche Diagnostics, Basel, Switzerland). Extracts were isolated by centrifugation at $4^{\circ} \mathrm{C}$ and $12,000 \times \mathrm{g}$ for $15 \mathrm{~min}$. Protein concentration was measured using the Bio-Rad Protein Assay reagent (Bio-Rad Laboratories, Hercules, CA, USA). Protein (10 $\mu \mathrm{g} /$ lane) were loaded and electrophoresis was conducted on $10 \%$ SDS-PAGE, and transferred to polyvinylidene difluoride membranes. The membranes were blocked in $5 \%$ bovine serum albumin (Bioshop Canada, Inc., Burlington, ON, Canada) in Tris-buffered saline containing $0.1 \%$ Tween-20 for $1 \mathrm{~h}$ at room temperature. The blocked membranes were incubated with primary antibodies overnight at $4^{\circ} \mathrm{C}$ with agitation, followed by incubation with a secondary antibody of horseradish peroxidase-conjugated goat anti-rabbit immunoglobulin $\mathrm{G}$ (1:3,000; cat. no. sc-2357; Santa Cruz Biotechnology, Inc., Dallas, TX, USA) for $1 \mathrm{~h}$ at room temperature. The blots were visualized using the Chemiluminescence Western Blot Detection System (BioSpectrum ${ }^{\circledR} 600$ Imaging System; UVP, LLC, Upland, CA, USA). Primary antibodies against phosphorylated (p)-c-Jun N-terminal kinase (JNK; 1:1,000, cat. no. 9251S), total (t)-JNK (1:1,000; cat. no. 9252S), p-focal adhesion kinase (FAK; 1:1,000; cat. no. 8556S), t-FAK (1:1,000; cat. no. 13009S), p-extracellular signal-regulated kinase (ERK)1/2 (1:3,000; cat. no. $9101 S)$ and t-ERK1/2 (1:3,000; cat. no. 9102S) were acquired from Cell Signaling Technology, Inc. (Danvers, MA, USA), and an antibody against $\alpha$-tubulin $(1: 1,000$; cat. no. sc-8035) was obtained from Santa Cruz Biotechnology, Inc.

Immunohistochemistry. For immunohistochemistry, mice were sacrificed at days 3 and 7 and tissues were harvested from normal and wounded areas. All tissues were fixed in $4 \%$ paraformaldehyde for at least $48 \mathrm{~h}$ at room temperature. Following fixation, tissues were dehydrated in graded ethanol from 70, 80, 90,95 to $100 \%$, cleared in xylene and embedded in paraffin. Sections $(4 \mu \mathrm{m})$ were mounted on glass slides at $45^{\circ} \mathrm{C}$, dewaxed using xylene, rehydrated with graded ethanol from 100, 95, 90, 80 and $70 \%$ to distilled water and stained at room temperature with hematoxylin and eosin (H\&E) or Masson's Trichrome. As part of the histological evaluation, all slides were examined by a pathologist without knowledge of treatments under a light microscope with magnification, $\mathrm{x} 20-100$. The area of collagen portion (\%) was measured using Image $\mathrm{J}$ software.

Statistical analysis. All experiments were repeated at least three times. Data analysis was performed with the Prism software (version 5; GraphPad Software, Inc., La Jolla, CA, USA). All data are presented as the mean \pm standard error of the mean. Statistical comparisons between two groups were determined using a two-tailed unpaired Student's t-test. Multiple comparisons were determined using one-way analysis of variance followed by Tukey's multiple comparison test. $\mathrm{P}<0.05$ was considered to indicate a statistically significant difference.

\section{Results}

Effect of PDRN size on wound closure in mice skin. Multiple previous studies have demonstrated that PDRNs promote wound healing in various animal models $(6,10,18-20)$. To 
evaluate the effect of PDRNs on wound healing, 8-week old hairless (SKH1) mice were given different sizes of PDRNs daily for a week. Based on previous results of in vivo pharmacological tests (21), $8 \mathrm{mg} / \mathrm{kg}$ doses of PDRNs were administered $(6,19)$. To evaluate wound closure, images were taken of PDRN-treated mouse skin at different healing stages (days 3 and 7). Administration of classic PDRN demonstrated no significant differences in wound closure or wound area compared with low- and high-PDRNs (Fig. 1).

Optimal PDRN improves the quality of wound closure in mice. Although differences in PDRN molecular weight did not affect the apparent surface wound closure, it did affect the quality of wound repairing. To analyze wound healing quality following treatment with PDRNs, the tissue repair process was monitored by H\&E staining. Among all the treatments, classic PDRN-treated mice demonstrated the best wound closure quality (Fig. 2). Although the wound closure was similar among the different groups, treatment with classic PDRN resulted in less lipid accumulation and a normal wound healing process. This result suggests that among the DNA mixtures, classic PDRN improved the quality of wound regeneration in mice the most.

Treatment with PDRN increases the collagen portion in wounded skin. The normal wound healing process primarily progresses based on the balance between collagen synthesis and collagen catabolism by matrix metalloproteinases (MMPs), and the adult wound bed predominantly contains type I collagen deposition during ECM remodeling (22). To determine whether classic PDRN affected collagen fiber accumulation, Masson's Trichrome staining was examined to analyze collagen composition. On day 3 , the collagen portion was significantly increased in classic PDRN-treated mouse skin compared with skin treated with other sizes of PDRN (Fig. 3A and B). At day 7, the majority of the PDRN-treated groups demonstrated similar collagen composition (Fig. 3C). However, compared with the other groups, the classic PDRN-treated group demonstrated increased collagen production and a decreased wound area; whereas, the other groups exhibited blood clots and were still in the early stages of wound healing. This data strongly supports the hypothesis that classic PDRN facilitates accumulation of collagen in earlier wound healing stages compared with other DNA mixtures.

Effect of PDRNs on migration in human fibroblasts. Although PDRN has been implicated in the process of repair and regeneration, the underlying mechanisms remain largely elusive. Wound healing involves cell migration and proliferation during wound closure. The mitogen-activated protein kinase (MAPK) pathway serves an important role in the regulation of cell migration and wound healing (23). Signaling by JNK is required for wounded epithelial cells to close the wound (24). Furthermore, extracellular signals stimulate cellular processes, including migration and proliferation, which are stimulated by FAK (25). To determine the mechanism underlying PDRN-mediated wound healing and determine whether it is mediated through the activation of FAK and JNK, FAK and JNK activation with vehicle and the different PDRN treatments $(50 \mu \mathrm{g} / \mathrm{ml})$ were examined (Fig. 4A). Classic PDRN induced the phosphorylation of JNK $24 \mathrm{~h}$ after treatment. FAK and ERK phosphorylation increased in the high and classic PDRN treatments compared with the vehicle treatment. However, the total forms additionally increased; FAK and ERK were not significantly activated by high and classic PDRN (Fig. 4B). These results suggest that classic PDRN activates JNK, which may be important for the classic PDRN-mediated wound healing process. To determine whether classic PDRN-induced wound closure was mediated by proliferation or migration, an in vitro scratch assay was conducted and subsequently incubated with EGF (a known promoter of wound healing), PDRNs or vehicle with mitomycin C, a known inhibitor of proliferation, for $24 \mathrm{~h}$. Images were captured from the initial point of cell migration at $0 \mathrm{~h}$ and at a scratch after $18 \mathrm{~h}$ (Fig. 4C). In the presence of vehicle, the wound area gradually closed and the rate of wound closure was significantly increased with EGF or classic PDRN treatment, suggesting that classic PDRN accelerated wound closure in human fibroblasts (Fig. 4D). EGF increased cell proliferation significantly compared with vehicle without mitomycin C; whereas, treatment with PDRNs did not affect proliferation (data not shown). This result suggests that classic PDRN may promote wound healing by increasing cell migration to attempt closure of the wound bed in human fibroblasts.

\section{Discussion}

Polydeoxyribonucleotide is a DNA mixture with molecular weights ranging between 50 and $1,500 \mathrm{kDa}$, produced by regulated purification and sterilization processes from Oncorhynchus mykiss (salmon trout) or Oncorhynchus keta (chum salmon) sperm DNA (9). Multiple previous studies have demonstrated that classic PDRN is associated with tissue repair, in addition to anti-ischemia and anti-inflammation responses (26-28). In the present study, it was investigated whether a diverse range of DNAs may exert similar effects under pathophysiological conditions. The effect of diversely sized DNAs (<50 kDa; 50-1,500 kDa; >1,500 kDa), including classic PDRN, has not been studied. The wound healing effect of differently sized PDRNs was examined and it was identified that classic PDRN stimulated positive factors associated with migration in the wound bed and a well ordered wound healing process.

A number of previous studies have demonstrated that classic PDRN functions as a growth promoter in the regeneration of various tissues, including wounded skin, and in osteoarthritis and ischemic-reperfusion injuries $(10,29,30)$. Based on the beneficial effects of PDRN, the effects of different molecular size DNA mixtures on wound healing were investigated. Epithelial cells begin to proliferate at the wound margin following injury, and epithelial migration may be subsequently triggered by growth factors and chemokines, including VEGF, PDGF and transforming growth factor- $\beta$ (31). In the wound-healing phase, granulation tissue begins the remodeling process via collagen, fibronectin and hyaluronic acid (31). It was identified that among the sizes examined, classic PDRN not only improved wound closure quality; however, additionally promoted the accumulation of collagen. The wound bed exhibited increased re-epithelialization subsequent to wounding, following treatment with classic PDRN compared with the other groups. 
$\mathbf{A}$

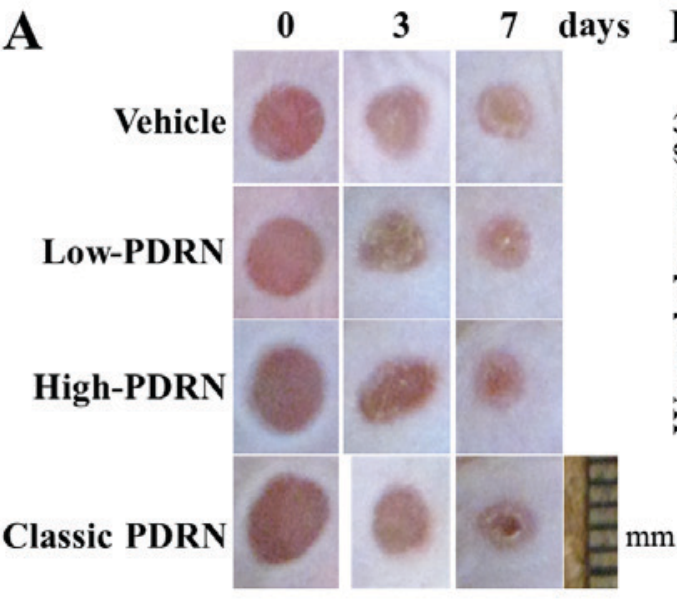

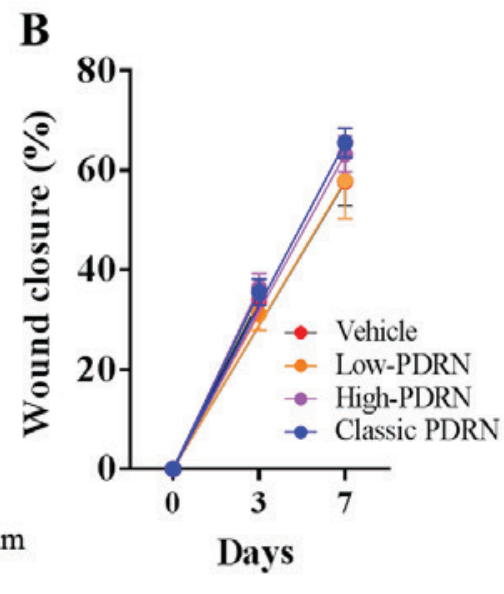

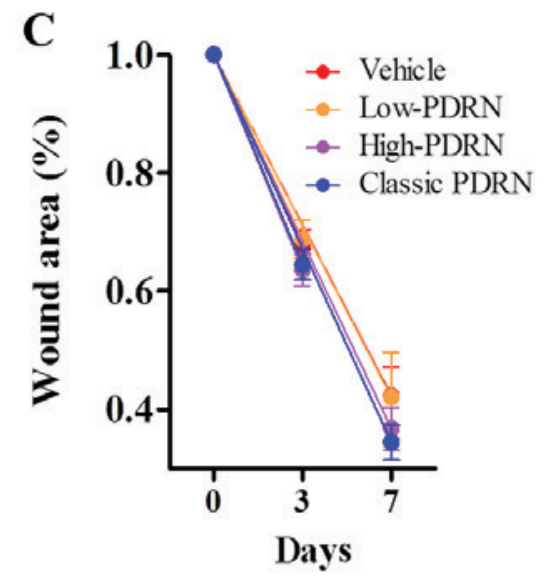

Figure 1. Effect of different sizes of PDRNs on wound closure in mice skin. (A) Representative images of skin wounds in male hairless mouse skin at different healing stages following treatment with PDRNs. Mice were randomly assigned to one of four groups: Vehicle, low (low-PDRN; <50 kDa), middle (classic PDRN; 50-1,500 kDa) and high (high-PDRN; >1,500 kDa) molecular weight PDRNs. Evaluated (B) wound-closure rates and (C) wound area in the four groups are expressed by the percentage of the initial (day 0 ) wound size. Results are presented as the mean \pm standard error of the mean ( $=8$ ). PDRNs, polydeoxyribonucleotides; classic PDRN, classic polydeoxyribonucleotide.
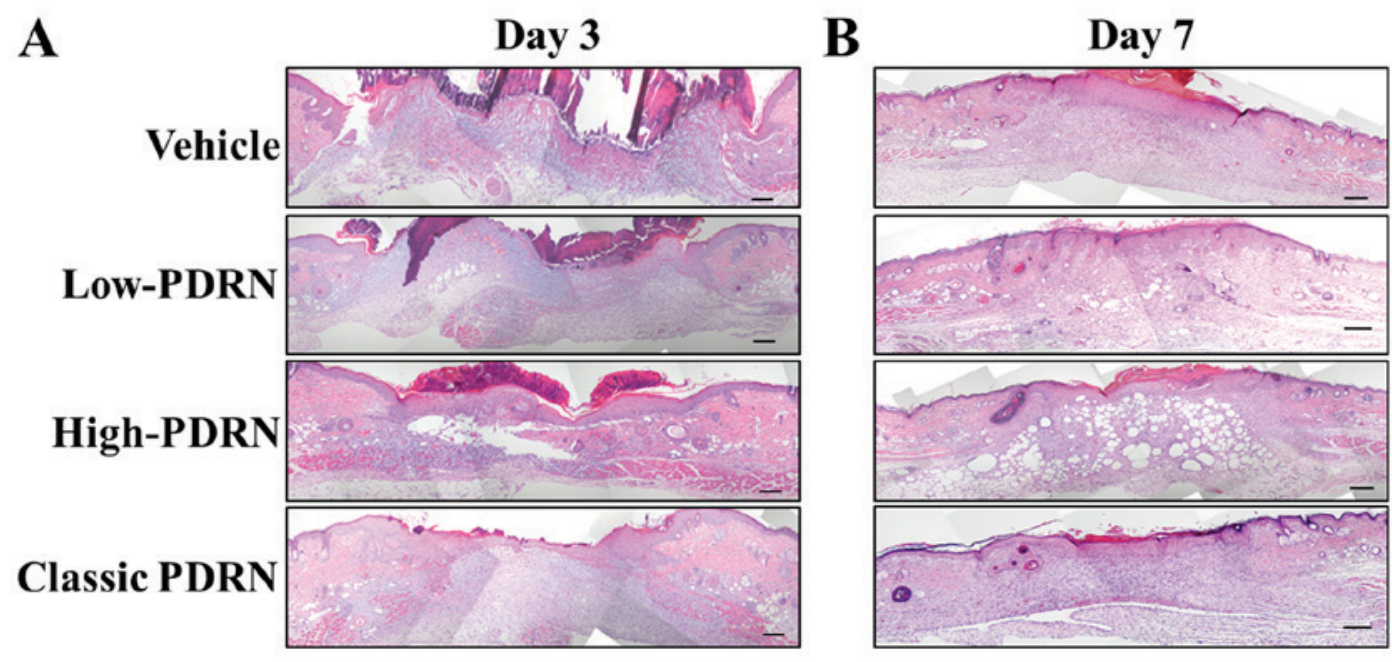

Figure 2. classic PDRN improves the quality of wound closure in mice. Healing process of 4 mm full-thickness excisional wounds in the skin of the back was evaluated at days (A) 3 and (B) 7 after treatment with PDRN. n=4/group and time point, four wounds per mouse. Histological sections of skin from the back of mice treated with different sizes of PDRNs. Scale bar, $200 \mu \mathrm{m}$. classic PDRN, classic polydeoxyribonucleotide; PDRNs, polydeoxyribonucleotides.

Furthermore, proliferation and migration promote the wound healing process. JNK, FAK and/or MAPK signaling promote cell proliferation and migration $(23-25,32)$. Promotion of cell motility is a well-known function of FAK. Treatment with PDRN induced an increase in the expression of the phosphorylated and total forms of FAK and ERK. Although there were no differences in the expression levels of activated FAK and ERK, PDRN may still regulate FAK and ERK expression. Treatment with EGF, a promoter of proliferation and migration, demonstrated phosphorylation of JNK and ERK. According to the present results, EGF stimulates JNK and ERK signaling to promote wound-healing processes in human fibroblasts. Notably, the observed increased JNK activity and wound closure effect with mitomycin $\mathrm{C}$ supports the hypothesis that the beneficial effects of classic PDRN on wound healing may be mediated by accelerating cell migration in human fibroblasts. Compared with low or high molecular weight PDRNs, middle range classic PDRN demonstrated better efficacy in wound healing processes. Numerous previous studies have reported that classic PDRN regulates cell proliferation, in addition to growth factor and pro-inflammatory signaling (33-35). Therefore, the wound healing ability of classic PDRN is not due to environmental improvement; however, rather more likely to be a result of numerous factors, including growth factors and chemokines during the wound healing process. JNK phosphorylation leads to transcription factors and target gene activation, including MMPs, and thereby promote wound healing (32). Although the present study demonstrated classic PDRN-mediated regulation of multiple signaling cascades, including total proteins of FAK and ERK, its downstream effectors and specific pathways linking to wound healing remain to be determined. In addition, how classic PDRN exerted pleotropic effects on the wound healing process requires further study. Therefore, future studies are required to examine the mechanism by which classic PDRN promotes wound healing via regulating multiple downstream effectors, including MMPs. 


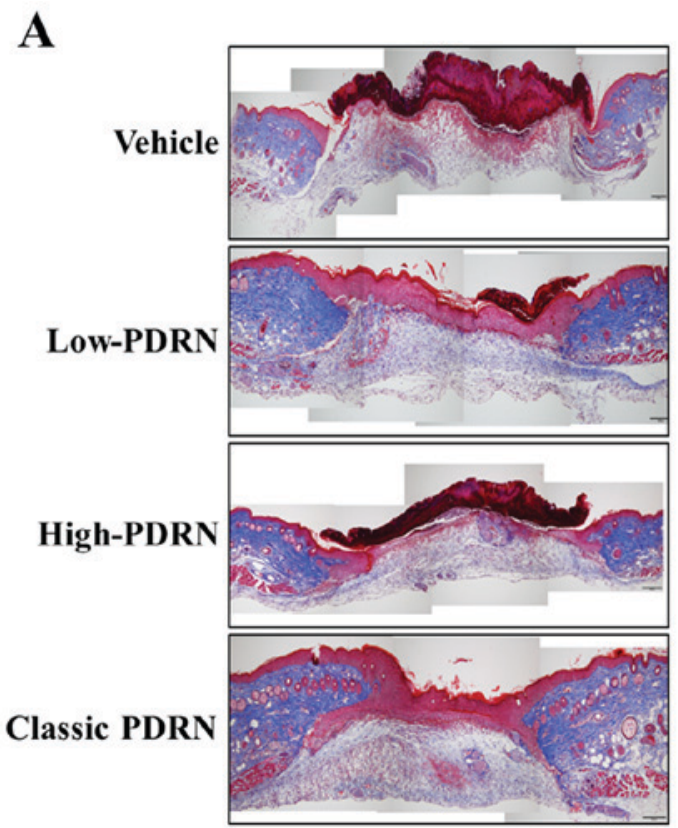

B

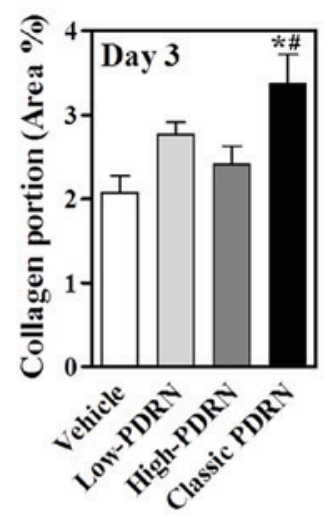

C

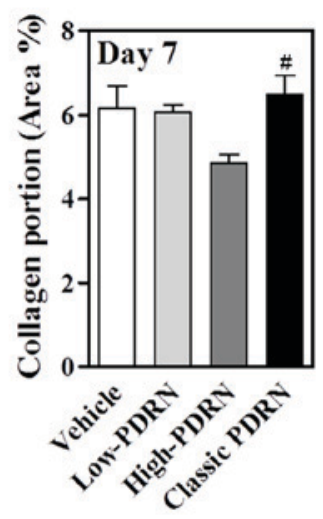

Figure 3. Treatment with PDRNs accelerated the collagen portion in wounded skin. (A) Masson Trichrome staining on day 3. Collagens stained blue were observed in the granulation tissue. Scale bar, $200 \mu \mathrm{m}$. Collagen portions (\%) at (B) day 3 and (C) day 7 are presented on the graph. Data are presented as the mean \pm standard error of the mean. $\mathrm{n}=4$ /group, one mouse with four wounds were analyzed. ${ }^{*} \mathrm{P}<0.05$ vs. vehicle. ${ }^{.} \mathrm{P}<0.05$ vs. high-PDRN. PDRNs, polydeoxyribonucleotides.

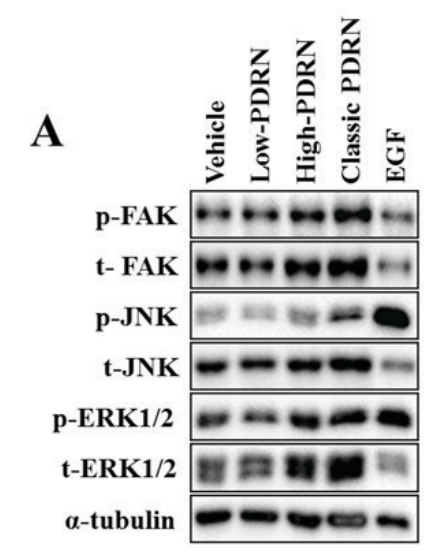

B

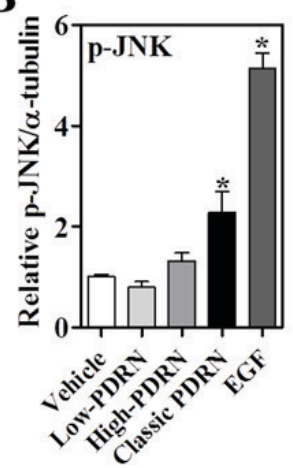

C

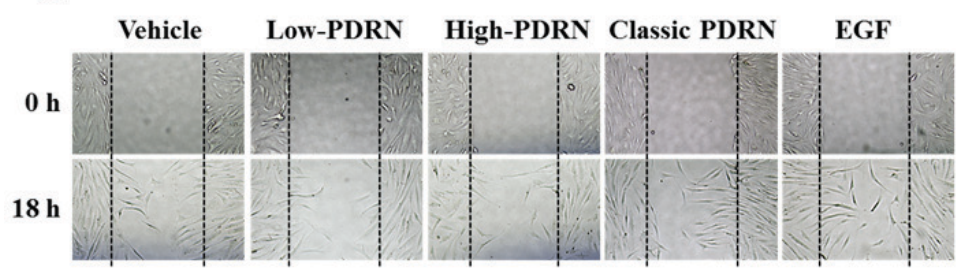

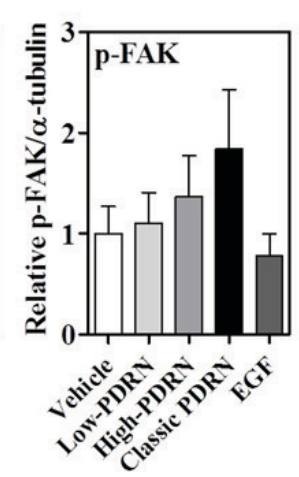

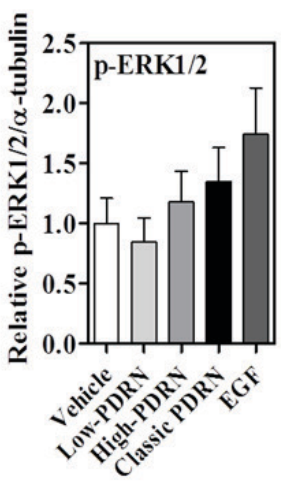

D

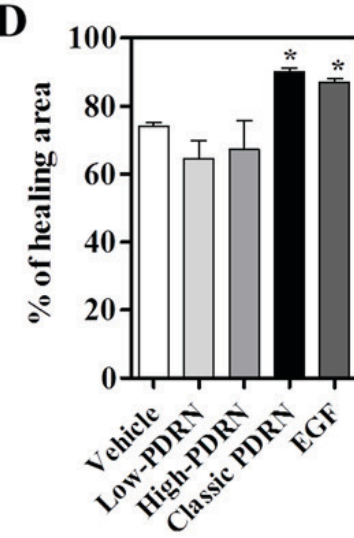

Figure 4. Effect of PDRNs on migration in human fibroblasts. (A) Human fibroblasts were treated with EGF and PDRNs for $24 \mathrm{~h}$ and subjected to western blotting with p-FAK, p-ERK, p-JNK, total form of FAK, ERK, JNK and $\alpha$-tubulin antibodies. (B) Quantitative densitometry analysis for p-JNK, p-FAK and p-ERK1/2 expression. Data are presented as the mean \pm standard error of the mean. "P $<0.05$ vs. vehicle. (C) Human fibroblasts were treated with vehicle, PDRNs $(50 \mu \mathrm{g} / \mathrm{ml})$ or EGF $(30 \mathrm{ng} / \mathrm{ml})$ following initial scratching to induce a wound. Representative images of wounds in human fibroblasts were taken at baseline $(0 \mathrm{~h})$ and $18 \mathrm{~h}$ later. (D) Quantified percentage of the wound healing area. Data are presented as the mean \pm standard error of the mean. "P<0.05 vs. vehicle. PDRNs, polydeoxyribonucleotides; classic PDRN, classic polydeoxyribonucleotide; EGF, epidermal growth factor; p, phosphorylated; t, total; FAK, focal adhesion kinase; ERK, extracellular signal-regulated kinase; JNK, c-Jun N-terminal kinase. 
In conclusion, all PDRNs are derived from the same DNA and may share the same biological effects on cell migration and proliferation. In the present study, it was demonstrated that a broad middle molecular size of PDRN ranging between $50-1,500 \mathrm{kDa}$ improved the quality of wound regeneration by promoting migration in a mouse skin wound model. Notably, classic PDRN-stimulating JNK signaling is critical for cell migration to improve the healing process. Collectively, the present results provide a novel perspective on the most effective molecular size of PDRN and the signaling for tissue repair, and suggest a novel potential drug to aid in the wound healing process.

\section{Acknowledgements}

All polydeoxyribonucleotide products were provided by Pharma Research Products Co., Ltd. (Pangyo, Korea).

\section{Funding}

The present study was supported by the Medical Research Center Program (grant no. 2017R1A5A2015369) and the Basic Science Research Program (grant nos.2015R1D1A1A01060454 and 2017R1D1A3B03031760) through the National Research Foundation of Korea.

\section{Availability of data and materials}

The datasets used and/or analyzed during the current study are available from the corresponding author on reasonable request.

\section{Authors' contributions}

KHH, JHK and EYP designed the study, conducted the experiments, analyzed the data and participated in writing the paper; $\mathrm{KHH}$ and EYP conducted and analyzed the in vivo wound healing experiment; JHK conducted and analyzed the in vitro experiments; SKC designed and supervised the entire project and wrote the final manuscript. All authors read, commented and approved the paper.

\section{Ethics approval and consent to participate}

The experimental protocol used in the present study, including the use of animals, was approved by the Institutional Animal Care and Use Committee, Yonsei University Wonju College of Medicine (Wonju, Korea; approval no. YWC-160504-1).

\section{Patient consent for publication}

Not applicable.

\section{Competing interests}

The authors declare that they have no competing interests.

\section{References}

1. Wahl SM, Wong H and McCartney-Francis N: Role of growth factors in inflammation and repair. J Cell Biochem 40: 193-199, 1989.
2. Greenhalgh DG, Sprugel KH, Murray MJ and Ross R: PDGF and FGF stimulate wound healing in the genetically diabetic mouse. Am J Pathol 136: 1235-1246, 1990.

3. Gilmore MA: Phases of wound healing. Dimens Oncol Nurs 5: 32-34, 1991.

4. Yu A, Niiyama H, Kondo S, Yamamoto A, Suzuki R and Kuroyanagi Y: Wound dressing composed of hyaluronic acid and collagen containing EGF or bFGF: Comparative culture study. J Biomater Sci Polym Ed 24: 1015-1026, 2013.

5. Tonello G, Daglio M, Zaccarelli N, Sottofattori E, Mazzei M and Balbi A: Characterization and quantitation of the active polynucleotide fraction (PDRN) from human placenta, a tissue repair stimulating agent. J Pharm Biomed Anal 14: 1555-1560, 1996.

6. Galeano M, Bitto A, Altavilla D, Minutoli L, Polito F, Calò M, Lo Cascio P, Stagno d'Alcontres F and Squadrito F: Polydeoxyribonucleotide stimulates angiogenesis and wound healing in the genetically diabetic mouse. Wound Repair Regen 16: 208-217, 2008.

7. Altavilla D, Squadrito F, Polito F, Irrera N, Calò M, Lo Cascio P, Galeano M, La Cava L, Minutoli L, Marini $\mathrm{H}$ and Bitto A: Activation of adenosine $\mathrm{A} 2 \mathrm{~A}$ receptors restores the altered cell-cycle machinery during impaired wound healing in genetically diabetic mice. Surgery 149: 253-261, 2011.

8. Bitto A, Polito F, Altavilla D, Minutoli L, Migliorato A and Squadrito F: Polydeoxyribonucleotide (PDRN) restores blood flow in an experimental model of peripheral artery occlusive disease. J Vasc Surg 48: 1292-1300, 2008.

9. Squadrito F, Bitto A, Irrera N, Pizzino G, Pallio G, Minutoli L and Altavilla D: Pharmacological activity and clinical use of PDRN. Front Pharmacol 8: 224, 2017.

10. Veronesi F, Dallari D, Sabbioni G, Carubbi C, Martini L and Fini M: Polydeoxyribonucleotides (PDRNs) from skin to musculoskeletal tissue regeneration via adenosine $\mathrm{A}_{2 \mathrm{~A}}$ receptor involvement. J Cell Physiol 232: 2299-2307, 2017.

11. Thellung S, Florio T, Maragliano A, Cattarini G and Schettini G: Polydeoxyribonucleotides enhance the proliferation of human skin fibroblasts: Involvement of A2 purinergic receptor subtypes. Life Sci 64: 1661-1674, 1999.

12. Valdatta L, Thione A, Mortarino C, Buoro M and Tuinder S: Evaluation of the efficacy of polydeoxyribonucleotides in the healing process of autologous skin graft donor sites: A pilot study. Curr Med Res Opin 20: 403-408, 2004.

13. Lee SH, Zheng Z, Kang JS, Kim DY, Oh SH and Cho SB: Therapeutic efficacy of autologous platelet-rich plasma and polydeoxyribonucleotide on female pattern hair loss. Wound Repair Regen 23: 30-36, 2015.

14. Yoon YC,LeeDH,Lee MY and YoonSH:Polydeoxyribonucleotide injection in the treatment of chronic supraspinatus tendinopathy: A case-controlled, retrospective, comparative study with 6-month follow-up. Arch Phys Med Rehabil 98: 874-880, 2017.

15. Squadrito F, Bitto A, Altavilla D, Arcoraci V, De Caridi G, De Feo ME, Corrao S, Pallio G, Sterrantino C, Minutoli L, et al: The effect of PDRN, an adenosine receptor A2A agonist, on the healing of chronic diabetic foot ulcers: Results of a clinical trial. J Clin Endocrinol Metab 99: E746-E753, 2014.

16. De Caridi G, Massara M, Acri I, Zavettieri S, Grande R, Butrico L, de Franciscis S and Serra R: Trophic effects of polynucleotides and hyaluronic acid in the healing of venous ulcers of the lower limbs: A clinical study. Int Wound J 13: 754-758, 2016.

17. Kim S, Kim J, Choi J, Jeong W and Kwon S: Polydeoxyribonucleotide improves peripheral tissue oxygenation and accelerates angiogenesis in diabetic foot ulcers. Arch Plast Surg 44: 482-489, 2017.

18. Jeong W, Yang CE, Roh TS, Kim JH, Lee JH and Lee WJ: Scar prevention and enhanced wound healing induced by polydeoxyribonucleotide in a rat incisional wound-healing model. Int J Mol Sci 18: pii: E1698, 2017.

19. Polito F, Bitto A, Galeano M, Irrera N, Marini H, Calò M, Squadrito F and Altavilla D: Polydeoxyribonucleotide restores blood flow in an experimental model of ischemic skin flaps. J Vasc Surg 55: 479-488, 2012.

20. Lee JH, Han JW, Byun JH, Lee WM, Kim MH and Wu WH: Comparison of wound healing effects between Oncorhynchus $k e t a$-derived polydeoxyribonucleotide (PDRN) and Oncorhynchus mykiss-derived PDRN. Arch Craniofac Surg 19: 20-34, 2018.

21. Jeon JW, Lee JI, Shin HP, Cha JM, Joo KR, Kim SH, Ko IG, Jin JJ, Kim SE and Kim CJ: Adenosine $\mathrm{A}_{2 \mathrm{~A}}$-receptor agonist polydeoxyribonucleotide promotes gastric ulcer healing in Mongolian gerbils. Anim Cells Syst 18: 399-406, 2014. 
22. Bohn G, Liden B, Schultz G, Yang Q and Gibson DJ: Ovine-based collagen matrix dressing: Next-generation collagen dressing for wound care. Adv Wound Care (New Rochelle) 5: 1-10, 2016.

23. Kajanne R, Miettinen P, Mehlem A, Leivonen SK, Birrer M, Foschi M, Kähäri VM and Leppä S: EGF-R regulates MMP function in fibroblasts through MAPK and AP-1 pathways. J Cell Physiol 212: 489-497, 2007.

24. Gazel A, Banno T, Walsh R and Blumenberg M: Inhibition of JNK promotes differentiation of epidermal keratinocytes. J Biol Chem 281: 20530-20541, 2006.

25. Mitra SK, Hanson DA and Schlaepfer DD: Focal adhesion kinase: in command and control of cell motility. Nat Rev Mol cell Biol 6: 56-68, 2005.

26. Jeong EK, Jang HJ, Kim SS, Lee SY, Oh MY, Kim HJ, Eom DW, Ham JY and Han DJ: Protective effect of polydeoxyribonucleotide against renal ischemia-reperfusion injury in mice. Transplant Proc 48: 1251-1257, 2016.

27. Kim JY, Pak CS, Park JH, Jeong JH and Heo CY: Effects of polydeoxyribonucleotide in the treatment of pressure ulcers. J Korean Med Sci 29 (Suppl 3): S222-S227, 2014.

28. Bitto A, Polito F, Irrera N, D'Ascola A, Avenoso A, Nastasi G, Campo GM, Micali A, Bagnato G, Minutoli L, et al: Polydeoxyribonucleotide reduces cytokine production and the severity of collagen-induced arthritis by stimulation of adenosine $\mathrm{A}\left({ }_{2} \mathrm{~A}\right)$ receptor. Arthritis Rheum 63: 3364-3371, 2011.
29. Buffoli B, Favero G, Borsani E, Boninsegna R, Sancassani G, Labanca M, Rezzani R, Nocini PF, Albanese M and Rodella LF: Sodium-DNA for bone tissue regeneration: An experimental study in rat calvaria. Biomed Res Int 2017: 7320953, 2017.

30. Noh TK, Chung BY, Kim SY, Kim SY, Lee MH, Kim MJ, Youn CS, Lee MW and Chang SE: Novel anti-melanogenesis properties of polydeoxyribonucleotide, a popular wound healing booster. Int J Mol Sci 17: 1448, 2016.

31. Bao P, Kodra A, Tomic-Canic M, Golinko MS, Ehrlich HP and Brem H: The role of vascular endothelial growth factor in wound healing. J Surg Res 153: 347-358, 2009.

32. Stevens LJ and Page-McCaw A: A secreted MMP is required for reepithelialization during wound healing. Mol Biol Cell 23: 1068-1079, 2012

33. Bitto A, Oteri G, Pisano M, Polito F, Irrera N, Minutoli L, Squadrito F and Altavilla D: Adenosine receptor stimulation by polynucleotides (PDRN) reduces inflammation in experimental periodontitis. J Clin Periodontol 40: 26-32, 2013.

34. Guizzardi S, Galli C, Govoni P, Boratto R, Cattarini G, Martini D, Belletti S and Scandroglio R: Polydeoxyribonucleotide (PDRN) promotes human osteoblast proliferation: A new proposal for bone tissue repair. Life Sci 73: 1973-1983, 2003.

35. Belletti S, Uggeri J, Gatti R, Govoni P and Guizzardi S: Polydeoxyribonucleotide promotes cyclobutane pyrimidine dimer repair in UVB-exposed dermal fibroblasts. Photodermatol Photoimmunol Photomed 23: 242-249, 2007. 